\title{
Gene Editing to the Rescue: Reversal of Social Deficits Associated with MECP2 Duplication
}

\author{
Zi-Xian $\mathrm{Yu}^{1,2,3} \cdot$ Dan-Yang Wang ${ }^{1,2,3} \cdot$ Xiao-Hong $\mathrm{Xu}^{1,3}{ }^{1}$
}

Received: 16 April 2020/Accepted: 9 May 2020/Published online: 29 May 2020

(C) Shanghai Institutes for Biological Sciences, CAS 2020

Methyl-CpG-binding protein 2 (MeCP2) is broadly recognized as the genetic cause of Rett Syndrome (RTT), a devastating neurodevelopmental disorder with the progressive loss in motor skills and speech that is found almost exclusively in young girls [1,2]. Over 95\% of RTT patients carry loss-of-function mutations in the X-linked MECP2 gene, while the same mutations in males largely lead to infantile death. Still, micro-duplications of the X chromosome segment that encompasses the $M E C P 2$ gene give rise to another neurological disorder known as MeCP2 duplication syndrome (MDS) that predominantly affects males, with symptoms including autistic features, seizures, profound intellectual disabilities, recurrent infections, hypotonia, and impaired social interactions. $\mathrm{MeCP} 2$ is expressed throughout the body with high levels in neurons, where it functions as a transcriptional repressor, a microRNA processor, and a regulator of RNA splicing within the nucleus to modulate gene expression, thereby regulating diverse aspects of neuronal function including fate determination, morphology, synaptogenesis, and synaptic plasticity. Indeed, neurons derived from the

Zi-Xian Yu and Dan-Yang Wang have contributed equally to this article.

Xiao-Hong Xu

xiaohong.xu@ion.ac.cn

1 Institute of Neuroscience, State Key Laboratory of Neuroscience, Chinese Academy of Sciences Center for Excellence in Brain Science and Intelligence Technology, Chinese Academy of Sciences, Shanghai 200031, China

2 University of the Chinese Academy of Sciences, Beijing 100049, China

3 Shanghai Center for Brain Science and Brain-Inspired Intelligence Technology, Shanghai 200031, China induced pluripotent stem cells of RTT or MDS patients exhibit altered dendritic morphology and spine density $[3,4]$.

Over the past two decades, transgenic animal models have been established to recapitulate the genetic defects of the MECP2 gene seen in RTT and MDS (null mutations or duplications), and have successfully reproduced many of the core behavioral symptoms found in patients. Remarkably, researchers have been able to demonstrate with the aid of genetic 'tricks' that systematically correcting $M E C P 2$ genetic abnormalities at the organism level in adult mice, either by re-expressing the $M E C P 2$ gene in an otherwise null background or by normalizing the expression level in duplication models, reverses the behavioral deficits after symptom onset [2]. These findings spark the hope that similar genetic correction procedures may also be beneficial in patients. Yet, as different cells likely contribute to distinct aspects of the behavioral deficits, the key question becomes which brain area/cell population to target in patients. In an exciting study published recently in Neuroscience Bulletin, Bin $\mathrm{Yu}$ and colleagues demonstrated that normalizing $\mathrm{MeCP} 2$ expression in the medial prefrontal cortex (mPFC) reverses the social deficits associated with $\mathrm{MeCP} 2$ duplication (Fig. 1) [5].

Using a transgenic mouse model of MDS, in which insertion of genomic sequences of the human version of the MECP2 (hMECP2) gene results in overexpression of MECP2, the authors first extensively characterized the behaviors of transgenic animals (males). Consistent with previous reports, they found that $M E C P 2$-transgenic (MECP2-Tg) males displayed heightened anxiety as well as potentiated fear learning and generalization. Moreover, MECP2-Tg males showed deficits in social recognition in that they had difficulty discriminating novel from familiar conspecifics (Fig. 1). Nevertheless, MECP2-Tg males were 


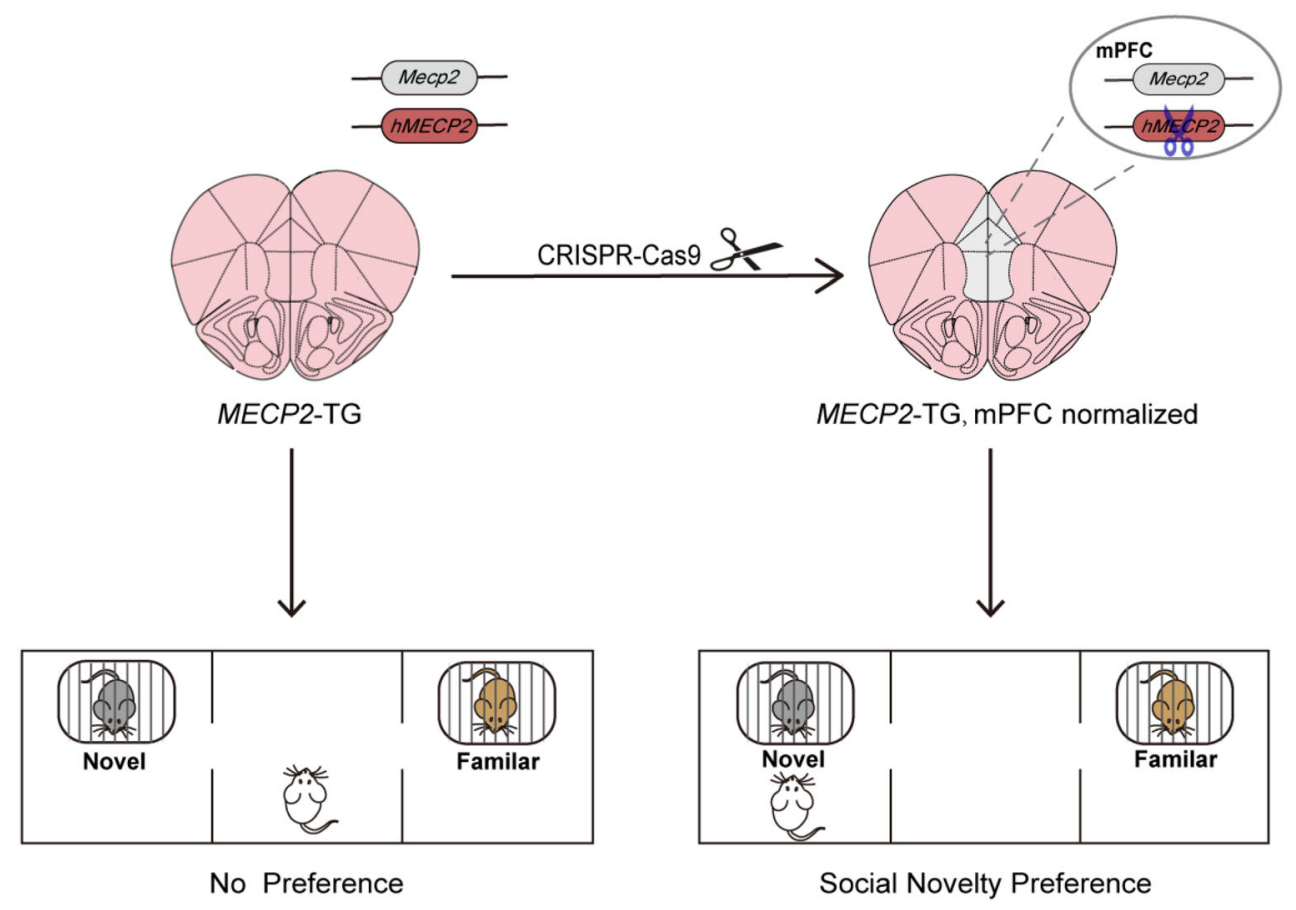

Fig. 1 Normalizing MeCP2 expression in the mPFC through a virusbased CRISPR-Cas 9 system rescues the social deficits associated with MeCP2 duplication. MECP2-TG animals positive for transgenes of the human $M E C P 2$ gene ( $M M E C P 2$ ) overexpress the MECP2 protein throughout the brain and show deficits in a social recognition test, in which they cannot discriminate between novel and familiar conspecifics and do not show a preference for novel conspecifics. Yu

normal in terms of sucrose consumption, cocaine-induced place preference, recognition of novel objects, and learning and remembering the location of a hidden escape box in a maze, demonstrating unimpaired reward processing and learning/memory capacity.

Next, to search for the neural substrates that correlate with the behavioral deficits, the authors performed restingstate fMRI analysis using a 9.4T scanner. Specifically, they measured the Regional Homogeneity (ReHo) value, which evaluates the similarity or synchronization of fMRI signals at a given voxel to those of its nearest neighbors by calculating Kendall's coefficient of concordance. MECP2$\mathrm{Tg}$ males had higher ReHo values in the $\mathrm{mPFC}$, anterior cingulate cortex, thalamus, and the retrosplenial and visual areas of cortex than control males, indicating higher neural activity/synchrony in these regions. Meanwhile, the ReHo values were lower in the nucleus accumbens, piriform cortex, bed nuclei of the stria terminalis (BST), hypothalamus, hippocampus, amygdala, and periaqueductal gray in $M E C P 2-T g$ males. Interestingly, these ReHo value differences were concentrated in areas implicated in the regulation of aversive and social behaviors, in line with the anxiety and social recognition deficits in $M E C P 2-T g$ males.

et al. used a virus-based CRISPR-Cas9 system to normalize MeCP2 expression in the $\mathrm{mPFC}$ by delivering single guide RNAs that specifically target, and thereby cause loss-of-function mutations, in the $h M E C P 2$ gene. MECP2-TG males with normalized $\mathrm{mPFC}$ $\mathrm{MeCP} 2$ expression now show preference for novel conspecifics, indicating rescue of the social recognition deficit.

Following these discoveries, the authors chose the $\mathrm{mPFC}$ as the target, since this region shows increased ReHo signals in MECP2-Tg males, and convergent evidence suggests that it plays a key role in social cognition. For example, lower gamma oscillation synchrony in the mPFC during social interactions but higher synchrony in a quiet state have been found in a transgenic mouse model of autism spectrum disorder (ASD) [6], which also shows social recognition deficits similar to $M E C P 2-T g$ males. To normalize MeCP2 expression, the authors cleverly used a virus-based CRISPR-Cas9 system that destroys the transgenic $h M E C P 2$ gene by delivering single guide RNA designed to specifically target the human but not the mouse $M E C P 2$ gene (Fig. 1). After virus injection, MECP2 expression in the $\mathrm{mPFC}$ was indeed decreased to the wild-type level in MECP2-Tg males. Even more excitingly, the social recognition deficits were also corrected in these males as they now showed a clear preference for novel conspecifics just like the wild-type controls (Fig. 1). By comparison, normalizing the mPFC MECP2 expression failed to rectify other behavioral abnormalities such as the increased anxiety and enhanced fear learning/memory. In addition, using the same viral strategy, the authors also normalized $\mathrm{MeCP} 2$ expression in the BST, a region that 
shows decreased ReHo signals in MECP2-Tg males and is known to regulate anxiety-related behaviors. Unfortunately, this manipulation did not result in any behavioral improvements, again highlighting the importance of the target site. Taken together, these results clearly demonstrate that normalizing $\mathrm{mPFC} \mathrm{MeCP} 2$ expression reverses social recognition deficits in a mouse model of MDS (Fig. 1).

Thus, Yu et al. have made an important step forward beyond the previous finding that showed whole-organism level correction of $M E C P 2$ gene can reverse behavioral deficits, by showing that region-specific correction of MeCP2 expression in the mPFC improves social cognition, at least in mice. It would be both interesting and informative in the future to test the relevance and effectiveness of the strategy used by $\mathrm{Yu}$ et al. in other animal models of MeCP2 disorders such as primate MeCP2-null or overexpression models. Thus, fMRI, as in $\mathrm{Yu}$ et al. could be first used to compare the whole-brain activity of $\mathrm{MeCP} 2$ transgenic animals with controls in different models to reveal candidate target sites [6-9]. Following this, a customized virus-based CRISPR-Cas9 system could be used to normalize MeCP2 levels in each site to determine whether different aspects of behavioral deficits could be rescued. Along this line, it would be imperative to subject animals to fMRI analysis again after MeCP2 expression is corrected. Such analysis will reveal whether the resting-state activity and the associated neural networks are also normalized in the targeted areas and whether these measurements predict or correlate with behavioral improvements. With such systematic efforts, normalizing MeCP2 expression via the CRISPR-Cas9 system may become a viable treatment option for RTT patients.

Furthermore, as social deficits are a core symptom in many other heritable psychiatric diseases such as ASD, for which there is also no treatment available, it will be interesting to determine whether gene-editing strategies coupled with neuroimaging studies such as that championed here by $\mathrm{Yu}$ et al. would provide an option to alleviate symptoms in these disorders. On this front, the rapidly evolving CRISPR-Cas9 field is providing ever-increasing possibilities to both turn up and turn down gene expression in vivo in a targeted manner [10]. In addition, newly developed CRISP-Cas9 systems could edit the genome with single-nucleotide precision in both the coding and regulatory regions of any gene, thereby correcting any de novo single-nucleotide mutations that might have caused a disease state. It is exciting to think that personalized treatments for psychiatric disorders using neuroimaging and gene-editing strategies may be a reality not too far ahead. In any case, Yu et al. have shown us a way forward.

\section{References}

1. Lombardi LM, Baker SA, Zoghbi HY. MECP2 disorders: from the clinic to mice and back. J Clin Invest 2015, 125: 2914-2923.

2. Qiu Z. Deciphering MECP2-associated disorders: disrupted circuits and the hope for repair. Curr Opin Neurobiol 2018, 48: 30-36.

3. Djuric U, Cheung AYL, Zhang W, Mok RS, Lai W, Piekna A, et al. MECP2e1 isoform mutation affects the form and function of neurons derived from Rett syndrome patient iPS cells. Neurobiol Dis 2015, 76: 37-45.

4. Nageshappa S, Carromeu C, Trujillo CA, Mesci P, EspunyCamacho I, Pasciuto E, et al. Altered neuronal network and rescue in a human MECP2 duplication model. Mol Psychiatry 2016, 21: 178-188.

5. Yu B, Yuan B, Dai JK, Cheng TL, Xia SN, He LJ, et al. Reversal of social recognition deficit in adult mice with MECP2 duplication via Normalization of MeCP2 in the medial prefrontal cortex. Neurosci Bull 2020. https://doi.org/10.1007/s12264-020-00467-w

6. Cao W, Lin S, Xia QQ, Du YL, Yang Q, Zhang MY, et al. Gamma oscillation dysfunction in $\mathrm{mPFC}$ leads to social deficits in neuroligin 3 R451C knockin mice. Neuron 2018, 97: $1253-1260$ e1257.

7. Qian A, Wang X, Liu H, Tao J, Zhou J, Ye Q, et al. Dopamine D4 receptor gene associated with the frontal-striatal-cerebellar loop in children with ADHD: A resting-state fMRI study. Neurosci Bull 2018, 34: 497-506.

8. Reddan MC, Wager TD. Modeling Pain Using fMRI: From regions to biomarkers. Neurosci Bull 2018, 34: 208-215.

9. Wang XH, Yu A, Zhu X, Yin H, Cui LB. Cardiopulmonary comorbidity, radiomics and machine learning, and therapeutic regimens for a cerebral fMRI predictor study in psychotic disorders. Neurosci Bull 2019, 35: 955-957.

10. Wang D, Zhang F, Gao G. CRISPR-Based therapeutic genome editing: Strategies and in vivo delivery by AAV vectors. Cell 2020, 181: 136-150. 